Bull. Austral. Math. Soc.

$11 \mathrm{~A} 25,20 \mathrm{D} 30,20 \mathrm{D} 40$

VoL. 75 (2007) [45-58]

\title{
ON ARITHMETIC FUNCTIONS OF FINITE GROUPS
}

\author{
Ashish Kumar DaS
}

The object of this paper is to develop and study group theoretic analogues of some of the fundamental concepts and results of arithmetic functions of positive integers.

\section{INTRODUCTION}

Arithmetic functions (that is, complex valued functions defined on positive integers) play a very important role not only in the theory of numbers but also in almost every branch of Mathematical sciences. Delsarte [4] and Cohen [3] have developed and studied group-theoretic analogues of arithmetic functions using finite Abelian groups, in place of positive integers, as the variables. However, most of their results do not not extend naturally to nonabelian groups. Recently, Leinster [6] developed and studied analogues of the 'sum-of-divisors' function for finite groups, especially for the nonabelian ones. Lucido and Pournaki [7], Mann [8], Marefat [9], Menegazzo [10], and many others have conducted detailed studies on various types of nontrivial group-theoretic arithmetic functions (of course, without calling them arithmetic functions).

Let $\mathcal{G}$ be the collection of all finite groups (up to isomorphism). Then $\mathcal{G}$ can be regarded as a monoid with respect to the direct product of groups (treating the isomorphic groups as the identical ones). The identity element of $\mathcal{G}$ is given by the trivial group $\{e\}$, the group of order 1 . Let $\mathcal{A}(\mathcal{G})$ denote the collection of all complex-valued functions with domain $\mathcal{G}$. Three very elementary yet very important members of $\mathcal{A}(\mathcal{G})$ are ||$, u$ and $\varepsilon$, given by

$$
|G|=\text { the order of } G, u(G)=1, \text { and } \varepsilon(G)= \begin{cases}1, & \text { if } G=\{e\} \\ 0, & \text { otherwise }\end{cases}
$$

where $G \in \mathcal{G}$. The object of this paper is to extend the ideas of Delsarte and Cohen to all finite groups (Abelian and nonabelian). Properly speaking, we study the structure of $\mathcal{A}(\mathcal{G})$ by developing group-theoretic analogues of some of the fundamental concepts and results of arithmetic functions (see [1]) of positive integers. We also try to characterise finite groups using analogues of divisor functions. It may be mentioned here that most

Received 5th July, 2006

Copyright Clearance Centre, Inc. Serial-fee code: 0004-9727/07 \$A2.00+0.00. 
of the group-theoretic analogues developed in this paper coincide, when restricted to the set of postive integers, with their number-theoretic counterparts; noting that the set of positive integers can be identified with the subset of $\mathcal{G}$ consisting of all finite cyclic groups under the injective map $n \mapsto C_{n}$ where $n$ is a positive integer and $C_{n}=\mathbb{Z} / n \mathbb{Z}$ is the cyclic group of order $n$.

\section{COPRIME gRoups, MULTIPLICATIVE FUNCTIONS, AND CONVOLUTIONS}

Let $G \in \mathcal{G}$ be a finite group. Then $G$ has a composition series given by $\{e\}=H_{0} \triangleleft H_{1} \triangleleft \cdots \triangleleft H_{n-1} \triangleleft H_{n}=G$. The set-with-multiplicities of composition factors associated to this composition series is given by $\mathcal{C}(G)=\left\{H_{i} / H_{i-1}: i=1,2, \ldots, n\right\}$ and it is uniquely determined by $G$ upto isomorphism of factors (see [5], Jordan-Hölder Theorem). By convention $\mathcal{C}(\{e\})=\emptyset$. It is a standard fact that $\forall K \unlhd G$ the set $\mathcal{C}(G)$ is the disjoint union (that is, union counting multiplicities) of the sets $\mathcal{C}(G / K)$ and $\mathcal{C}(K)$.

We shall say (see [6]) that the groups $G_{1}, G_{2} \in \mathcal{G}$ are coprime or relatively prime if $\mathcal{C}\left(G_{1}\right)$ and $\mathcal{C}\left(G_{2}\right)$ have no member (that is, composition factor) in common; in case $\mathcal{C}\left(G_{1}\right)$ and $\mathcal{C}\left(G_{2}\right)$ have no Abelian member in common then $G_{1}$ and $G_{2}$ will be called almost coprime. Thus, the groups $G_{1}$ and $G_{2}$ will be coprime whenever they have coprime orders, and if $G_{1}$ and $G_{2}$ are Abelian then the converse is also true. It may be mentioned here that the alternating groups $A_{5}$ and $A_{6}$ are coprime but they do not have coprime orders.

A function $f \in \mathcal{A}(\mathcal{G})$ which is not identically zero will be called multiplicative if we have $f\left(G_{1} \times G_{2}\right)=f\left(G_{1}\right) f\left(G_{2}\right)$ whenever $G_{1}, G_{2} \in \mathcal{G}$ are coprime; in case $f\left(G_{1} \times G_{2}\right)$ $=f\left(G_{1}\right) f\left(G_{2}\right)$ holds for all $G_{1}, G_{2} \in \mathcal{G}$ then $f$ will be called completely multiplicative. Further, we shall say that the function $f$ is almost completely multiplicative if we have $f\left(G_{1} \times G_{2}\right)=f\left(G_{1}\right) f\left(G_{2}\right)$ whenever $G_{1}, G_{2} \in \mathcal{G}$ are almost coprime. Thus, we have $f(\{e\})=1$ if $f$ satisfies any of the multiplicativity conditions. It is easy to see that || , $u$ and $\varepsilon$ are all completely multiplicative functions.

Given $G \in \mathcal{G}$, let $\Pi \mathcal{C}(G)$ denote the direct product of all members (counting multiplicities) of the set-with-multiplicities $\mathcal{C}(G)$. As a convention, we take $\Pi \mathcal{C}\{e\}=\{e\}$. If $f$ and $g$ are any two functions in $\mathcal{A}(\mathcal{G})$ then we shall define their convolution to be the function $f * g \in \mathcal{A}(\mathcal{G})$ given by

$$
(f * g)(G)=\sum f(H) g(K)
$$

where $G \in \mathcal{G}$, and the summation is over all ordered pairs $(H, K) \in \mathcal{G} \times \mathcal{G}$ which satisfy one of the following conditions:

(i) One of $H$ and $K$ is $G$, and the other is $\{e\}$,

(ii) $H \times K=\Pi C(G)$, and none of $H$ and $K$ is $\{e\}$. 
REMARK 2.1. If $f, g \in \mathcal{A}(\mathcal{G})$ then one can also define their ordinary product $f g \in \mathcal{A}(\mathcal{G})$ given by $f g(G)=f(G) g(G) \forall G \in \mathcal{G}$. However, the convolution defined above turns out to be more fruitful.

PROPOSITION 2.2. $\mathcal{A}(\mathcal{G})$ is a commutative ring with identity $\varepsilon$ under the additive and the multiplicative operations given respectively by ordinary addition and convolution of functions.

Proof: Note that if $f, g, h \in \mathcal{A}(\mathcal{G})$ then

$$
((f * g) * h)(G)=(f *(g * h))(G)=\sum f(L) g(M) h(N),
$$

where $G \in \mathcal{G}$, and the summation is over all ordered triples $(L, M, N) \in \mathcal{G} \times \mathcal{G} \times \mathcal{G}$ which satisfy one of the following conditions:

(i) One of $L, M$ and $N$ is $G$, and the other two are $\{e\}$,

(ii) $L \times M \times N=\Pi \mathcal{C}(G)$, and no two of $L, M$ and $N$ are $\{e\}$.

The associative law for multiplication is thus established. The other properties can be easily proved.

REMARK 2.3. In general, the convolution of two multiplicative functions in $\mathcal{A}(\mathcal{G})$ is not multiplicative. However, if the groups $G_{1}, G_{2} \in \mathcal{G}$ are coprime as well as completely reducible then $(f * g)\left(G_{1} \times G_{2}\right)=(f * g)\left(G_{1}\right)(f * g)\left(G_{2}\right)$; noting that on the completely reducible groups the convolution mentioned above coincides with the 'direct convolution' which is multiplicative (see [3]).

Consider the factorisation map $\rho: \mathcal{G} \longrightarrow \mathcal{G}$ given by $\rho(G)=\Pi \mathcal{C}(G)$ where $G \in \mathcal{G}$. By Jordan-Hölder Theorem, $\rho$ is well-defined. Clearly, $\rho(G)=G$ if and only if $\mathrm{G}$ is completely reducible in $\mathcal{G}$. It is also easy to see that $\rho$ is a monoid homomorphism and it preserves 'coprimeness' of groups in $\mathcal{G}$. thus, if $f \in \mathcal{A}(\mathcal{G})$ is multiplicative then $f \circ \rho \in \mathcal{A}(\mathcal{G})$ is also multiplicative. In fact, we have

PROPOSITION 2.4. If $f, g \in \mathcal{A}(\mathcal{G})$ are multiplicative then $(f * g) \circ \rho \in \mathcal{A}(\mathcal{G})$ is also multiplicative.

Proof: Follows from Remark 2.3.

From the definition of convolution, it follows that $\rho$ is distributive over convolution. More precisely, we have

Proposition 2.5. If $f, g \in \mathcal{A}(\mathcal{G})$ then $(f * g) \circ \rho=(f \circ \rho) *(g \circ \rho)$.

As an immediate consequence we have

Corollary 2.6. $\rho$ induces a unitary ring homomorphism of $\mathcal{A}(\mathcal{G})$ into itself given by $f \mapsto f \circ \rho$ where $f \in \mathcal{A}(\mathcal{G})$.

As in the case of the ring of arithemetic functions of positive integers, the ring $\mathcal{A}(\mathcal{G})$ also has invertible elements. 
Proposition 2.7. If $f \in \mathcal{A}(\mathcal{G})$ with $f(\{e\}) \neq 0$ then there is a unique $g \in \mathcal{A}(\mathcal{G})$ such that $f * g=g * f=\varepsilon$.

Proof: We shall show that, for each $G \in \mathcal{G}$, the equation $f * g(G)=\varepsilon(G)$ has a unique solution for $g(G)$.

If $G=\{e\}$, then the equation $f * g(\{e\})=\varepsilon(\{e\})=1$ has a unique solution given by

$$
g(\{e\})=\frac{1}{f(\{e\})} .
$$

So, let $G \in \mathcal{G}$ be nontrivial, and assume that for each $K \in \mathcal{G}$, with $|K|<|G|$, the equation $f * g(K)=\varepsilon(K)$ has a unique solution for $g(K)$. Now, the equation $f * g(G)=\varepsilon(G)=0$ can be written as

$$
f(\{e\}) g(G)=-\sum f(H) g(K)
$$

where the summation is over all ordered pairs $(H, K) \in \mathcal{G} \times \mathcal{G}$ such that either $H=G$ and $K=\{e\}$, or $H \times K=\Pi \mathcal{C}(G)$ and none of $H$ and $K$ is $\{e\}$. Clearly each such $K$ satisfies $|K|<|G|$, and so by induction hypothesis $g(G)$ is uniquely determined, namely,

$$
g(G)=\frac{-1}{f(\{e\})} \sum f(H) g(K),
$$

This completes the proof.

The function $g$ mentioned in the above proposition will be called the inverse of the function $f$, and will be denoted by $f^{-1}$.

COROLLARY 2.8. The set of all functions $f \in \mathcal{A}(\mathcal{G})$ with $f(\{e\}) \neq 0$ forms an Abelian group under the operation given by convolution.

Proof: Let $f, g \in \mathcal{A}(\mathcal{G})$ with $f(\{e\}) \neq 0$. Then $g(\{e\}) \neq 0$ if and only if $(f * g)(\{e\})=f(\{e\}) g(\{e\}) \neq 0$. Hence, in view of Proposition 2.2, the corollary follows.

Returning back to multipliplicative functions in $\mathcal{A}(\mathcal{G})$ we have

Proposition 2.9. If $f, g \in \mathcal{A}(\mathcal{G})$ are such that $f \circ \rho$ and $(f * g) \circ \rho$ are multiplicative then $g \circ \rho$ is also multiplicative.

Proof: Let $G_{1}, G_{2} \in \mathcal{G}$ be any two coprime groups. We shall use induction on the direct factors of $\rho\left(G_{1}\right)$ and $\rho\left(G_{2}\right)$ to prove that $(g \circ \rho)\left(G_{1} \times G_{2}\right)=(g \circ \rho)\left(G_{1}\right)(g \circ \rho)\left(G_{2}\right)$

If $G_{1}=G_{2}=\{e\}$ then

$$
1=((f * g) \circ \rho)(\{e\})=(f \circ \rho)(\{e\})(g \circ \rho)(\{e\}),
$$

and so, as the induction hypothesis, we assume that for each direct factor $K_{1}$ of $\rho\left(G_{1}\right)=\Pi\left(G_{1}\right)$ and for each direct factor $K_{2}$ of $\rho\left(G_{2}\right)=\Pi\left(G_{2}\right)$ with $K_{1} \times K_{2}$ $\neq \rho\left(G_{1}\right) \times \rho\left(G_{2}\right)$ we have

$$
(g \circ \rho)\left(K_{1} \times K_{2}\right)=(g \circ \rho)\left(K_{1}\right)(g \circ \rho)\left(K_{2}\right)
$$


that is, $g\left(K_{1} \times K_{2}\right)=g\left(K_{1}\right) g\left(K_{2}\right)$. Then, since $(f * g) \circ \rho$ is multiplicative, we have

$$
\begin{array}{rlrl}
((f * g) \circ \rho)\left(G_{1} \times G_{2}\right) & =((f * g) \circ \rho)\left(G_{1}\right)((f * g) \circ \rho)\left(G_{2}\right) \\
& \text { or } \quad & \sum f(H) g(K) & =\left(\sum f\left(H_{1}\right) g\left(K_{1}\right)\right)\left(\sum f\left(H_{2}\right) g\left(K_{2}\right)\right) \\
& \text { or } \quad \sum H_{1} \quad \sum\left(H_{1} \times H_{2}\right) g\left(K_{1} \times K_{2}\right) & =\sum f\left(H_{1} \times H_{2}\right) g\left(K_{1}\right) g\left(K_{2}\right),
\end{array}
$$

where the summations are over all ordered pairs $(H, K),\left(H_{1}, K_{1}\right),\left(H_{2}, K_{2}\right) \in \mathcal{G} \times \mathcal{G}$ such that $H \times K=\rho\left(G_{1}\right) \times \rho\left(G_{2}\right), H_{1} \times K_{1}=\rho\left(G_{1}\right)$, and $H_{2} \times K_{2}=\rho\left(G_{2}\right)$. Therefore, using the induction hypothesis and the fact that $(f \circ \rho)(\{e\})=1$, we have

$$
g\left(\rho\left(G_{1}\right) \times \rho\left(G_{2}\right)\right)=g\left(\rho\left(G_{1}\right)\right) g\left(\rho\left(G_{2}\right)\right) .
$$

This completes the proof.

COROLlaRY 2.10. The set of all multiplicative functions in $\mathcal{A}(\mathcal{G})$ of the form $f \circ \rho$, where $f \in \mathcal{A}(\mathcal{G})$, is an Abelian group under the operation given by convolution.

Proof: It is easy to see that the set under consideration is same as the set of all functions in $\mathcal{A}(\mathcal{G})$ of the form $f \circ \rho$, where $f \in \mathcal{A}(\mathcal{G})$ is a multiplicative function; noting that $f \circ \rho=(f \circ \rho) \circ \rho$. We shall in fact show that this set is a subgroup of the Abelian group considered in Corollary 2.8 .

Let $f, g \in \mathcal{A}(\mathcal{G})$ be any two multiplicative functions. Then, by Proposition 2.4, $(f \circ \rho) *(g \circ \rho)=(f * g) \circ \rho$ is multiplicative. Also, we have

$$
(f \circ \rho) *\left(f^{-1} \circ \rho\right)=\left(f * f^{-1}\right) \circ \rho=\varepsilon \circ \rho=\varepsilon .
$$

So, $(f \circ \rho)^{-1}=f^{-1} \circ \rho$, and, by the above proposition, $f^{-1} \circ \rho$ is multiplicative. Hence, the corollary follows.

\section{The Möbius Function ANd the RELATEd RESUlts}

The Möbius function, which is one of the most useful examples of arithmetic functions of positive integers, is given by

$$
\mu(n)= \begin{cases}(-1)^{\omega(n)}, & \text { if } \omega(n)=\Omega(n) \\ 0, & \text { otherwise }\end{cases}
$$

where $n$ is a positive integer, $\omega(n)$ is the number of distinct prime factors of $n$, and $\Omega(n)$ is the number of prime factors (counting multiplicity) of $n$.

We define the group-theoretic analogues of $\omega, \Omega$ and the Möbius function $\mu$ as

$$
\begin{aligned}
& \omega(G)=\text { number of distinct (that is, non-isomorphic) factors in } \mathcal{C}(G), \\
& \Omega(G)=\text { number of factors (counting multiplicity) in } \mathcal{C}(G), \text { and } \\
& \mu(G)= \begin{cases}(-1)^{\omega(G),} & \text { if } \omega(G)=\Omega(G) \\
0, & \text { otherwise }\end{cases}
\end{aligned}
$$


where $G \in \mathcal{G}$. Note that $\omega(\{e\})=0=\Omega(\{e\})$ and so $\mu(\{e\})=1$.

Considering $G$ to be $C_{2} \times C_{2}$ and $C_{4}$, one can see that the Möbius function $\mu(G)$ defined above is not an extension of the Möbius functions defined by Delsarte and Cohen. However, taking $G=C_{n}$, we have

$$
\omega\left(C_{n}\right)=\omega(n), \Omega\left(C_{n}\right)=\Omega(n), \text { and } \mu\left(C_{n}\right)=\mu(n)
$$

because $C_{n}=C_{p_{1} r_{1}} \times \cdots \times C_{p_{k}{ }^{r_{k}}}$, where $p_{1}{ }^{r_{1}}, \ldots, p_{k}{ }^{{ }{ }_{k}}$ is the standard prime factorisation of $n$, and so $\mathcal{C}\left(C_{n}\right)$ consists of the simple groups $C_{p_{i}}$, each having multiplicity $r_{i}, 1 \leqslant i \leqslant k$.

LEMmA 3.1. $\Omega\left(G_{1} \times G_{2}\right)=\Omega\left(G_{1}\right)+\Omega\left(G_{2}\right)$ for all $G_{1}, G_{2} \in \mathcal{G}$, and $\omega\left(G_{1} \times G_{2}\right)$ $=\omega\left(G_{1}\right)+\omega\left(G_{2}\right)$ if $G_{1}$ and $G_{2}$ are coprime.

Proof: Given $G_{1}, G_{2} \in \mathcal{G}$, we know that $\mathcal{C}\left(G_{1} \times G_{2}\right)$ is the disjoint union (that is, union counting multiplicities) of $\mathcal{C}\left(G_{1}\right)$ and $\mathcal{C}\left(G_{2}\right)$. Hence the lemma follows.

Proposition 3.2. The Möbius function $\mu \in \mathcal{A}(\mathcal{G})$ is multiplicative, and $\mu *$ $u=u * \mu=\varepsilon$ that is, $\mu^{-1}=u$ and $u^{-1}=\mu$.

Proof: The first part follows from Lemma 3.1. For the second part, one notes that $\mu \circ \rho=\mu$ and $u \circ \rho=u$ where $\rho$ is the factorisation map considered in section 2. So, by Propositions 2.4 and $2.5, \mu * u$ is multiplicative and $(\mu * u)(G)=(\mu * u)(\Pi \mathcal{C}(G))$ $\forall G \in \mathcal{G}$. Now, for $G \neq\{e\}, \Pi \mathcal{C}(G)$ is a product of powers of distinct simple groups in $\mathcal{G}$, and

$$
\begin{aligned}
(\mu * u)\left(P^{k}\right) & =\mu(\{e\})+\mu(P)+\mu\left(P^{2}\right)+\cdots+\mu\left(P^{k}\right) \\
& =1-1+0+\cdots+0=0
\end{aligned}
$$

for each simple group $P \in \mathcal{G}$, for each positive integer $k$. So, it follows that $(\mu * u)(G)$ $=(u * \mu)(G)=\varepsilon(G) \forall G \in \mathcal{G}$.

As an immediate consequence, we have the following analogue of the Möbius inversion formula.

Proposition 3.3. For $f, g \in \mathcal{A}(\mathcal{G})$,

$$
f=g * u \Leftrightarrow g=f * \mu \text {. }
$$

Proof: We have to simply 'multiply' the first equation on the right by $\mu$ to get the second, and the second equation on the right by $u$ to get the first.

An important example of a completely multiplicative function of positive integers is the Liouville's function given by

$$
\lambda(n)=(-1)^{\Omega(n)}
$$

where $n$ is a positive integer. We define the group-theoretic analogues of $\lambda$ as

$$
\lambda(G)=(-1)^{\Omega(G)}
$$

where $G \in \mathcal{G}$. 
Proposition 3.4. The Liouville's function $\lambda \in \mathcal{A}(\mathcal{G})$ is completely multiplicative, and $\lambda * \mu^{2}=\mu^{2} * \lambda=\varepsilon$ that is, $\lambda^{-1}=\mu^{2}$ where $\mu^{2}$ is the ordinary product of $\mu$ with itself. Also, for each $G \in \mathcal{G}$,

$$
(\lambda * u)(G)=(u * \lambda)(G)= \begin{cases}1, & \text { if } \rho(G)=\rho(H \times H) \text { for some } H \in \mathcal{G} \\ 0, & \text { otherwise. }\end{cases}
$$

ProOF: The first assertion follows from Lemma 3.1. The remaining assertions are proved using an argument similar to the one that was used to prove the second part of Proposition 3.2 , except that in this case we have

$$
\left(\lambda * \mu^{2}\right)\left(P^{k}\right)=\lambda\left(P^{k}\right)+\lambda\left(P^{k-1}\right)=(-1)^{k}+(-1)^{k-1}=0,
$$

and

$$
\begin{aligned}
(\lambda * u)\left(P^{k}\right) & =\lambda(\{e\})+\lambda(P)+\lambda\left(P^{2}\right)+\cdots+\lambda\left(P^{k}\right) \\
& =1-1+1-\cdots+(-1)^{k}= \begin{cases}0, & \text { if } k \text { is odd } \\
1, & \text { if } k \text { is even }\end{cases}
\end{aligned}
$$

for each simple group $P \in \mathcal{G}$, and for each positive integer $k$. Hence, it follows that if $G \in \mathcal{G}$ then $\left(\lambda * \mu^{2}\right)(G)=\left(\mu^{2} * \lambda\right)(G)=\varepsilon(G)$, and also $(\lambda * u)(G)=0$ or 1 depending on whether there exists or does not exist a factor in $\mathcal{C}(G)$ having odd multiplicity.

In Proposition 2.7, we have seen, in particular, how to compute the inverse of a multiplicative function on $\mathcal{G}$. However, for completely multiplicative functions of the form $f \circ \rho$ the computation of the inverse is easy.

Proposition 3.5. Let $f \in \mathcal{A}(\mathcal{G})$ be a multiplicative function such that $f=f \circ \rho$. Then $f$ is completely multiplicative if and only if $f *(\mu f)=(\mu f) * f=\varepsilon$ that is, $f^{-1}=\mu f$ where $\mu f$ is the ordinary product of $\mu$ and $f$.

Proof: For each $G \in \mathcal{G}$, we have

$$
\begin{aligned}
(f *(\mu f))(G) & =\sum f(H) \mu(K) f(K)=\sum f(H \times K) \mu(K) \\
& =f(G)(u * \mu)(G)=\varepsilon(G)
\end{aligned}
$$

by proposition 3.2; noting that $(f \circ p)(G)=f(G), f(\{e\})=1$ and $\varepsilon(G)=0$ for $G \neq\{e\}$.

Conversely, since $f$ is mulptiplicative it is enough to show that $f\left(P^{k}\right)=f(P)^{k}$ for each simple group $P$ in $\mathcal{G}$ and for each positive integer $k$. Now,

$$
\begin{aligned}
(\mu f) * f=\varepsilon & \Longrightarrow((\mu f) * f)\left(P^{k}\right)=0 \\
& \Longrightarrow \mu(\{e\}) f(\{e\}) f\left(P^{k}\right)+\mu(P) f(P) f\left(P^{k-1}\right)=0 \\
& \Longrightarrow f\left(P^{k}\right)=f(P) f\left(P^{k-1}\right)
\end{aligned}
$$

and so, by iteration, $f\left(P^{k}\right)=f(P)^{k}$. This completes the proof. 


\section{DIVISOR FUNCTIONS AND THEIR MULTIPLICATIVITY}

Some well-known examples of divisor functions which form an integral part of arithmetic functions of positive integers are given by

$$
\tau(n)=\sum_{d \mid n} 1, \quad \sigma(n)=\sum_{d \mid n} d, \quad \text { and } \quad \sigma_{\alpha}(n)=\sum_{d \mid n} d^{\alpha}
$$

where $n$ is a positive integer and $\alpha$ is any complex number.

Taking cue from [6], we define the group-theoretic anlogues of these functions as

$$
\tau(G)=\sum_{N \unlhd G} 1, \quad \sigma(G)=\sum_{N \unlhd G}|N|, \quad \text { and } \quad \sigma_{\alpha}(G)=\sum_{N \unlhd G}|N|^{\alpha}
$$

where $G \in \mathcal{G}$. Thus, $\tau(G)$ is the number of normal subgroups of $G, \sigma(G)$ is the sum of the orders of normal subgroups of $G$, and $\sigma_{\alpha}(G)$ the sum of the $\alpha^{\text {th }}$ powers of the orders of normal subgroups of $G$.

Clearly, $\sigma_{0}(G)=\tau(G)$ and $\sigma_{1}(G)=\sigma(G)$. Also, in particular, if we take $G=C_{n}$ then $\sigma_{\alpha}\left(C_{n}\right)=\sigma_{\alpha}(n)$; noting that there is an one to one correspondence between the normal subgroups of $C_{n}$ and the divisors of $n$ given by $N \mapsto|N|$, where $N \unlhd C_{n}$.

Since every proper non-trivial normal subgroup $N$ of a finite group $G$ satisfies $2 \leqslant|N| \leqslant|G| / 2$, we have

$$
2 \tau(G)+|G|-3 \leqslant \sigma(G) \leqslant 1+\tau(G) \frac{|G|}{2} .
$$

PROPOSITION 4.1. If $G \in \mathcal{G}$ then $\sigma(G)=\sum_{g \in G} \tau\left(G / N_{g}\right)$ where $N_{g}$ is the smallest normal subgroup of $G$ containing $g$.

Proof: We know that, $\forall g \in G$, the normal subgroups of $G / N_{g}$ are in one to one correspondence with the set $\left\{N: N_{g} \unlhd N \unlhd G\right\}=\{N: g \in N \unlhd G\}$, and so $\tau\left(G / N_{g}\right)=|\{N: g \in N \unlhd G\}|$. Therefore,

$$
\begin{aligned}
\sigma(G) & =\sum_{N \unlhd G}|N| \quad=\sum_{N \unlhd G} \sum_{g \in N} 1=\sum_{N \unlhd G} \sum_{g \in G} \delta_{g, N}, \\
& =\sum_{g \in G} \sum_{N \unlhd G} \delta_{g, N}=\sum_{g \in G} \sum_{g \in N \unlhd G} 1=\sum_{g \in G} \tau\left(G / N_{g}\right) ;
\end{aligned}
$$

here $\delta_{g, N}=1$ or 0 , according as $g \in N$ or $g \notin N$.

As a corollary we have the following number theoretic identity.

COROLlary 4.2. For any positive integer $n$,

$$
\sigma(n)=\sum_{k=0}^{n-1} \tau(\operatorname{gcd}(n, k))
$$


Proof: Putting $G=C_{n}$, in the above proposition, we have

$$
\begin{aligned}
\sigma(n)=\sigma\left(C_{n}\right) & =\sum_{k=0}^{n-1} \tau\left(\frac{C_{n}}{\langle k\rangle}\right), \quad\langle k\rangle \text { is the subgroup of } C_{n} \text { generated by } k \\
& =\sum_{k=0}^{n-1} \tau\left(\frac{C_{n}}{C_{k^{\prime}}}\right), \quad \text { where } k^{\prime}=\frac{n}{\operatorname{gcd}(n, k)} \\
& =\sum_{k=0}^{n-1} \tau\left(C_{\operatorname{gcd}(n, k)}\right)=\sum_{k=0}^{n-1} \tau(\operatorname{gcd}(n, k)) .
\end{aligned}
$$

Given any two groups $G_{1}$ and $G_{2}$ (finite or infinite, Abelian or non-abelian), we now develop a condition which is equivalent to saying that every normal subgroup of the product $G_{1} \times G_{2}$ is of the form $N_{1} \times N_{2}$ with $N_{i} \unlhd G_{i}, i=1,2$.

We shall say that the groups $G_{1}$ and $G_{2}$ have a subgroup in common if there exist non-trivial subgroups $H_{1}$ of $G_{1}$, and $H_{2}$ of $G_{2}$ such that $H_{1} \cong H_{2}$.

THEOREM 4.3. Let $G_{1}$ and $G_{2}$ be any two groups. Then the following conditions are equivalent:

(1) Every normal subgroup of the product $G_{1} \times G_{2}$ is of the form $N_{1} \times N_{2}$ with $N_{1} \unlhd G_{1}$ and $N_{2} \unlhd G_{2}$.

(2) For each $H_{1} \triangleleft G_{1}$ and for each $H_{2} \triangleleft G_{2}$, the centres $Z\left(G_{1} / H_{1}\right)$ and $Z\left(G_{2} / H_{2}\right)$ of the quotient groups $G_{1} / H_{1}$ and $G_{2} / H_{2}$ have no subgroup in common.

Proof: Suppose that $G_{1}$ and $G_{2}$ satisfy the second condition. Let $N \unlhd G_{1} \times G_{2}$. Set $H_{1}=\pi_{1}\left(\left(G_{1} \times\left\{e_{2}\right\}\right) \cap N\right)$ and $H_{2}=\pi_{2}\left(\left(\left\{e_{1}\right\} \times G_{2}\right) \cap N\right)$ where $e_{i}$ are identities of $G_{i}$ and $\pi_{i}: G_{1} \times G_{2} \longrightarrow G_{i}$ are projections, $i=1,2$. Then

$$
H_{1} \times\left\{e_{2}\right\},\left\{e_{1}\right\} \times H_{2} \subset N \subset \pi_{1}(N) \times \pi_{2}(N)
$$

and so

$$
H_{1} \times H_{2}=\left(H_{1} \times\left\{e_{2}\right\}\right)\left(\left\{e_{1}\right\} \times H_{2}\right) \subset \pi_{1}(N) \times \pi_{2}(N)
$$

It may be noted here that $H_{i} \unlhd G_{i}$ and $H_{i} \unlhd \pi_{i}(N), i=1,2$. Now, suppose $a_{1} \in \pi_{1}(N)$. Then $\left(a_{1}, a_{2}\right) \in N$ for some $a_{2} \in G_{2}$; in fact $a_{2} \in \pi_{2}(N)$. Therefore, $\forall g_{1} \in G_{1}$, we have

$$
\begin{aligned}
\left(g_{1} a_{1} g_{1}^{-1}, a_{2}\right) & =\left(g_{1}, e_{2}\right)\left(a_{1}, a_{2}\right)\left(g_{1}{ }^{-1}, e_{2}\right) \in N \\
& \Longrightarrow\left(g_{1} a_{1} g_{1}^{-1} a_{1}^{-1}, e_{2}\right) \in N \\
& \Longrightarrow g_{1} a_{1} g_{1}^{-1} a_{1}^{-1} \in H_{1} \\
& \Longrightarrow g_{1} H_{1} a_{1} H_{1}=a_{1} H_{1} g_{1} H_{1} \in G_{1} .
\end{aligned}
$$


Thus, $a_{1} H_{1} \in Z\left(G_{1} / H_{1}\right)$. So, we have $\pi_{1}(N) / H_{1} \subset Z\left(G_{1} / H_{1}\right)$. Similarly, $\pi_{2}(N) / H_{2} \subset Z\left(G_{2} / H_{2}\right)$. Note that if $a_{1}, b_{1} \in \pi_{1}(N)$ then $\left(a_{1}, a_{2}\right),\left(b_{1}, b_{2}\right) \in N$ for some $a_{2}, b_{2} \in \pi_{2}(N)$, and so $\left(a_{1} b_{1}^{-1}, a_{2} b_{2}^{-1}\right),\left(a_{1} b_{1}, a_{2} b_{2}\right) \in N$. Therefore,

$$
\begin{gathered}
a_{1} H_{1}=b_{1} H_{1} \Longleftrightarrow a_{1} b_{1}^{-1} \in H_{1} \Longleftrightarrow\left(a_{1} b_{1}^{-1}, e_{2}\right) \in N \\
\Longleftrightarrow\left(e_{1}, a_{2} b_{2}^{-1}\right) \in N \Longleftrightarrow a_{2} b_{2}^{-1} \in H_{2} \Longleftrightarrow a_{2} H_{2}=b_{2} H_{2} .
\end{gathered}
$$

This means that we have a well-defined injective map $f: \pi_{1}(N) / H_{1} \longrightarrow \pi_{2}(N) / H_{2}$ given by $f\left(a_{1} H_{1}\right)=a_{2} H_{2}$ where $\left(a_{1}, a_{2}\right) \in N$. Also,

$$
f\left(a_{1} H_{1} b_{1} H_{1}\right)=f\left(a_{1} b_{1} H_{1}\right)=a_{2} b_{2} H_{2}=a_{2} H_{2} b_{2} H_{2}=f\left(a_{1} H_{1}\right) f\left(b_{1} H_{1}\right),
$$

showing that $f$ is a homomorphism. Finally, if $b \in \pi_{2}(N)$ then $(a, b) \in N$ for some $a \in \pi_{1}(N)$ and so $f\left(a H_{1}\right)=b H_{2}$, which implies that $f$ is surjective. Thus $f$ is an isomorphism. Hence it follows from the hypothesis that $\pi_{i}(N) / H_{i}$ are trivial subgroups of $Z\left(G_{i} / H_{i}\right), i=1,2$. Therefore, $H_{i}=\pi_{i}(N), i=1,2$, and so $N=H_{1} \times H_{2}$, by ((4.4)).

Conversely, suppose $G_{1}$ and $G_{2}$ do not satisfy the second condition. So, there exist $H_{i} \triangleleft G_{2}, i=1,2$, such that $Z\left(G_{1} / H_{1}\right)$ and $Z\left(G_{2} / H_{2}\right)$ have a subgroup in common. Let $K_{i} / H_{i}$ be non-trivial subgroups of $Z\left(G_{i} / H_{i}\right), i=1,2$, such that there is an isomorphism $F: K_{1} / H_{1} \longrightarrow K_{2} / H_{2}$. Put

$$
N=\left\{\left(a_{1}, a_{2}\right) \in K_{1} \times K_{2}: F\left(a_{1} H_{1}\right)=a_{2} H_{2}\right\} .
$$

Let $\left(a_{1}, a_{2}\right),\left(b_{1}, b_{2}\right) \in N$ then $F\left(a_{1} H_{1}\right)=a_{2} H_{2}$ and $F\left(b_{1} H_{1}\right)=b_{2} H_{2}$. So, $F\left(a_{1} b_{1}^{-1} H_{1}\right)$ $=a_{2} b_{2}{ }^{-1} H_{2}$. Thus

$$
\left(a_{1}, a_{2}\right)\left(b_{1}, b_{2}\right)^{-1}=\left(a_{1} b_{1}^{-1}, a_{2} b_{2}^{-1}\right) \in N,
$$

showing that $N$ is a subgroup of $G_{1} \times G_{2}$. Again let $\left(a_{1}, a_{2}\right) \in N$ and $\left(g_{1}, g_{2}\right) \in G_{1} \times G_{2}$. Then,

$$
\left(g_{1}, g_{2}\right)\left(a_{1}, a_{2}\right)\left(g_{1}, g_{2}\right)^{-1}=\left(g_{1} a_{1} g_{1}^{-1}, g_{2} a_{2} g_{2}^{-1}\right) \in K_{1} \times K_{2},
$$

since $K_{i} \unlhd G_{i}, i=1,2$. Also, since

$$
a_{i} H_{i} \in K_{i} / H_{i} \subset Z\left(G_{i} / H_{i}\right), i=1,2,
$$

we have

$$
F\left(g_{1} a_{1} g_{1}^{-1} H_{1}\right)=F\left(a_{1} H_{1}\right)=a_{2} H_{2}=g_{2} a_{2} g_{2}{ }^{-1} H_{2} .
$$

Thus $\left(g_{1}, g_{2}\right)\left(a_{1}, a_{2}\right)\left(g_{1}, g_{2}\right)^{-1} \in N$, and so $N \unlhd G_{1} \times G_{2}$. On the other hand, suppose $N$ is of standard form $N_{1} \times N_{2}$ where $N_{i} \unlhd G_{i}, i=1,2$. Then, $\pi_{i}(N)=N_{i}, i=1,2$. But since $\mathrm{F}$ is bijective, we have $\pi_{i}(N)=K_{i}, i=1,2$. Therefore, $N=K_{1} \times K_{2}$. Since $K_{1} / H_{1}$ is non-trivial, there is some $a_{1} \in K_{1}$ such that $a_{1} H_{1} \neq H_{1}$. But $\left(a_{1}, e_{2}\right) \in K_{1} \times K_{2}=N$. So, $F\left(a_{1} H_{1}\right)=e_{2} H_{2}=H_{2}$, the zero element of $K_{2} / H_{2}$. Therefore, since $F$ is injective, we have $a_{1} H_{1}=H_{1}$, the zero element of $K_{1} / H_{1}$. This contradiction shows that $N$ is not of the form mentioned in the first condition.

The following corollary generalises [6, Proposition 3.3]. 
CoROLlaRY 4.4. If $G_{1}, G_{2} \in \mathcal{G}$ are almost coprime then every normal subgroup of the product $G_{1} \times G_{2}$ is of the form $N_{1} \times N_{2}$ with $N_{1} \unlhd G_{1}$ and $N_{2} \unlhd G_{2}$.

PROOF: Let $H_{1} \triangleleft G_{1}$ and $H_{2} \triangleleft G_{2}$ be such that the centres $Z\left(G_{1} / H_{1}\right)$ and $Z\left(G_{2} / H_{2}\right)$ have a subgroup in common. So, there are non-trivial subgroups $K_{i} / H_{i}$ of $Z\left(G_{i} / H_{i}\right), i=1,2$, such that $K_{1} / H_{1} \cong K_{2} / H_{2}$. Then $\mathcal{C}\left(K_{1} / H_{1}\right)=\mathcal{C}\left(K_{2} / H_{2}\right)$. Since $H_{i} \triangleleft K_{i} \unlhd G_{i}$, we have $\mathcal{C}\left(K_{i} / H_{i}\right) \subseteq \mathcal{C}\left(K_{i}\right) \subseteq \mathcal{C}\left(G_{i}\right), i=1,2$. Thus, $\mathcal{C}\left(G_{1}\right)$ and $\mathcal{C}\left(G_{2}\right)$ have an Abelian member in common.

By considering $G_{1}=S_{4}, G_{2}=C_{3}$, and noting that the symmetric group $S_{4}$ and the quotient $S_{4} / V \cong S_{3}$ have trivial centres, one can easily see that the converse of the above corollary is not true; $V$ is the normal subgroup $\{e,(12)(34),(13)(24),(14)(23)\}$ of $S_{4}$. However, as an immediate consequence of the above corollary we have

Proposition 4.5. If $f \in \mathcal{A}(\mathcal{G})$ is multiplicative (or, almost completely multiplicative) then the function $F \in \mathcal{A}(\mathcal{G})$ given by

$$
F(G)=\sum_{N \unlhd G} f(N)
$$

is also multiplicative (or, almost completely multiplicative).

Proof: Let $G_{1}, G_{2} \in \mathcal{G}$ be a pair of coprime (or, almost coprime) groups. Clearly, if $N_{1} \unlhd G_{1}$ and $N_{2} \unlhd G_{2}$ then $N_{1}$ and $N_{2}$ are also coprime (or, almost coprime). So, we have

$$
\begin{aligned}
F\left(G_{1} \times G_{2}\right) & =\sum_{N \unlhd\left(G_{1} \times G_{2}\right)} f(N)=\sum_{N_{1} \unlhd G_{1}, N_{2} \unlhd G_{2}} f\left(N_{1} \times N_{2}\right) \\
& =\sum_{N_{1} \unlhd G_{1}} \sum_{N_{2} \unlhd G_{2}} f\left(N_{1}\right) f\left(N_{2}\right)=F\left(G_{1}\right) F\left(G_{2}\right) .
\end{aligned}
$$

COROLlary 4.6. $\sigma_{\alpha}$ is almost completely multiplicative.

Proof: It is enough to note that $G \mapsto|G|^{\alpha}$, for $G \in \mathcal{G}$, defines a completely multiplicative function in $\mathcal{A}(\mathcal{G})$.

\section{ChaRACTERISATION OF GROUPS USING DIVISOR FUNCTIONS}

Most trivial characterisation of groups using divisor functions is perhaps the following:

$$
\sigma_{\alpha}(G)=|G|^{\alpha}+1 \Longleftrightarrow G \text { is a simple group }
$$

where $G \in \mathcal{G}$, and $\alpha$ is a complex number. Also, we know that any finite Abelian group $G$ has a (normal) subgroup of order $d$ for every divisor $d$ of $|G|$, and $G$ has exactly one such subgroup for every divisor $d$ if and only if $G$ is cyclic. Therefore, for any Abelian group $G \in \mathcal{G}$, we have $\sigma_{\alpha}(G) \geqslant \sigma_{\alpha}(|G|)$, and the equality holds if and only if $G$ is cyclic. 
Lemma 5.1. Let $G \in \mathcal{G}$ be such that $G=\bigcup_{N \triangleleft G} N$. Then $\tau(G) \geqslant 5$.

Proof: Since $|N| \leqslant|G| / 2 \quad \forall N \triangleleft G$, and since the identity element of $G$ is a common member of all normal subgroups of $G$, it follows that any two proper normal subgroups of $G$ can contain at the most $|G|-1$ distinct elements. Hence $G$ must have atleast three proper nontrivial normal subgroups, which in turn implies that $\tau(G) \geqslant 5$.

Proposition 5.2. Let $G \in \mathcal{G}$. If $\sigma(G) \leqslant 2|G|+2$ then $G \neq \bigcup_{N \triangleleft G} N$.

Proof: Let us assume that $G=\bigcup_{N \triangleleft G} N$. Then, for each $g \in G$, the smallest normal subgroup $N_{g}$ of $G$ containing $g$ is a proper normal subgroup of $G$, and so $G / N_{g} \neq\{e\}$ which means $\tau\left(G / N_{g}\right) \geqslant 2$. Therefore, by Proposition 4.1, we have

$$
\begin{aligned}
\sigma(G) & =\sum_{g \in G} \tau\left(G / N_{g}\right)=\tau(G)+\sum_{g \in G, g \neq e} \tau\left(G / N_{g}\right) \\
& \geqslant \tau(G)+2(|G|-1) .
\end{aligned}
$$

Since $\sigma(G) \leqslant 2|G|+2$, it follows that $\tau(G) \leqslant 4$ which contrdicts the Lemma 5.1. Hence the proposition follows.

From [2, Theorem 1], we know that if $G \in \mathcal{G}$ is such that $G \neq \bigcup_{N \triangleleft G} N$ then every Abelian quotient of $G$ (that is, every quotient of $G$ which is Abelian) is cyclic. Hence, we have the following corollary to the above proposition, which is also an improvement to the 'Abelian Quotient Theorem' proved in [6]:

COROLlary 5.3. If $G$ is a finite group with $\sigma(G) \leqslant 2|G|+2$ then every Abelian quotient of $G$ is cyclic.

REMARK 5.4. If $q: G \longrightarrow G^{\prime}$ is a homomorphism of groups $G, G^{\prime} \in \mathcal{G}$ then $\tau(q(G))$ $\leqslant \tau(G)$, since $q^{-1}(M) \unlhd G \forall M \unlhd q(G)$. In particular, we have $\tau(G / N) \leqslant \tau(G) \quad \forall$ $G \in \mathcal{G}$ and $\forall N \unlhd G$; moreover, the inequality is strict if $N$ is nontrivial.

Proposition 5.5. Let $G \in \mathcal{G}$ be such that $\tau(G)=5$ and $G=\bigcup_{N \triangleleft G} N$. Then $G=C_{2} \times C_{2}$.

Proof: By ([2, Theorem 1]), there is a normal subgroup of $G$ such that $G / N$ $=C_{p} \times C_{p}$ for some prime $p$. So,

$$
\begin{aligned}
\tau(G / N)=\tau\left(C_{p} \times C_{p}\right)=p+3 & \Longrightarrow \tau(G) \geqslant p+3, \text { since } \tau(G) \geqslant \tau(G / N), \\
& \Longrightarrow p=2 \Longrightarrow N=\{e\},
\end{aligned}
$$

otherwise $5=\tau(G)>\tau(G / N)=p+3=5$ which is absurd. Hence the proposition follows.

The Proposition 5.5 tells us that if $G \neq C_{2} \times C_{2}$ then the hypothesis of the Corollary 5.3 can be further improved to $\sigma(G) \leqslant 2|G|+3$. 
In [6], Leinster has studied the groups $G \in \mathcal{G}$ which satisfy the condition $\sigma(G)$ $=2|G|$, and he termed such groups as 'perfect groups' (not to be confused with the ones which are more standard and mean something else). He proved that an Abelian group $G \in \mathcal{G}$ is perfect if and only if $G=C_{n}$ where $n$ is a perfect number that is, $\sigma(n)=2 n$, and he conjectured that there are infinitely many nonabelian perfect groups. However, it requires some effort to find examples of nonabelian perfect groups. Leinster has exhibited only three examples of such groups, namely,

$$
S_{3} \times C_{5}, A_{5} \times C_{61} \times C_{31} \times C_{8}, A_{6} \times C_{361} \times C_{127} \times C_{8} .
$$

We mention below few more examples of nonabelian perfect groups:

Consider the generalised quaternion group $\mathbb{Q}_{4 m}$ of order $4 m, m \geqslant 2$, given by

$$
\mathbb{Q}_{4 m}=\left\langle a, b \mid a^{2 m}=1, b^{2}=a^{m}, b a b^{-1}=a^{-1}\right\rangle .
$$

It can be easily proved that if $m$ is odd then the proper normal subgroups of $\mathbb{Q}_{4 m}$ are precisely the subgroups of the cyclic group generated by $a$. Therefore, $\sigma\left(\mathbb{Q}_{4 m}\right)$ $=4 m+\sigma(2 m)$ if $m$ is odd. Using multiplicativity of $\sigma$ one can see that the nonabelian group $\mathbb{Q}_{12}, \mathbb{Q}_{20} \times C_{19}, \mathbb{Q}_{28} \times C_{13}, \mathbb{Q}_{244} \times A_{5} \times C_{43} \times C_{11}$ and $\mathbb{Q}_{220} \times C_{109}$ are all perfect groups in the sense of Leinster.

\section{REFERENCES}

[1] T.M. Apostol, Introduction to analytic number theory, Springer International Student Edition (Narosa Publishing House, New Delhi, 1993).

[2] M.A. Brodie, R.F. Chamberlain and L.-C. Kappe, 'Finite coverings by normal subgroups', Proc. Amer. Math. Soc. 104 (1988), 669-674.

[3] E. Cohen, 'Arithemetical functions of finite abelian groups', Math. Ann. 142 (1961), $165-182$.

[4] P.S. Delsarte, 'Fonctions de Möbius sur les groupes abeliens finis', Ann. of Math. 49 (1948), 600-609.

[5] N. Jacobson, Basic algebra, vol I (W.H. Freeman and Company, U.S.A., 1974).

[6] T. Leinster, 'Perfect number and groups', arXiv :math.GR/0104012 v1 Apr 2001.

[7] M.S. Lucido and M.R. Pournaki, 'Elements with square roots in finite groups', Algebra Colloq. 12 (2005), 677-690.

[8] A. Mann, 'Finite groups containing many involutions', Proc. Amer. Math. Soc. 122 (1994), $383-326$.

[9] Y. Marefat, 'Conjugacy structure type and degree structure type in finite p-groups', Turkish J. Math. 24 (2000), 321-326.

[10] F. Menegazzo, 'The number of generators of a finite group', Irish Math. Soc. Bull. 50 (2003), 117-128. 
Department of Mathematics

North Eastern Hill University

Permanent Campus

Shillong-793022, Meghalaya

India

e-mail: akdas@nehu.ac.in 